\title{
POTENCIAIS INTERAÇÕES MEDICAMENTOSAS EM PACIENTES PORTADORES DE DOENÇA RENAL CRÔNICAEM TRATAMENTO DE HEMODIÁLISE
}

Anielly Almeida ROCHA ${ }^{1}$

Josilene Dália ALVES ${ }^{2}$

Fernanda Regina Casagrande Giachini Vitorino GIACHINI ${ }^{3}$

${ }^{1}$ Discente do curso de Bacharelado em Farmácia. Universidade Federal de Mato Grosso (UFMT). anny_rocha@hotmail.com.

${ }^{2}$ Enfermeira.Mestre em imunologia e parasitologia básicas e aplicadas. Instituto Federal de Mato Grosso (IFMT). josydalia@ hotmail.com

${ }^{3}$ Farmacêutica Bioquímica, Docente da Universidade Federal de Mato Grosso (UFMT). fernandagiachini@hotmail.com

Recebido em: 20/12/2016 - Aprovado em: 15/08/2017 - Disponibilizado em: 30/12/2017

\begin{abstract}
RESUMO
INTRODUÇÃO: O paciente portador de doença renal crônica (DRC) faz uso de uma ampla gama de medicamentos, potencializando a ocorrência de possíveis interações medicamentosas (IM)s. OBJETIVO: Identificar potencias IMs nos prontuários dos pacientes portadores de DRC do estágio 5, em tratamento de hemodiálise, classificando-as quanto a sua gravidade e separando a sua ocorrência quanto as co-morbidades de cada paciente. METODOLOGIA:Foram analisados os prontuários dos pacientes portadores de DRC de estágio 5, que estavam em tratamento de hemodiálise no município de Barra do Garças MT. Para identificar as possíveis IMs foi consultado o MICROMEDEX ${ }^{\circledR}$, software que possui um sistema interativo para checar sua ocorrência. RESULTADOS: Foram analisados o prontuário de 49 pacientes, com faixa etária entre 25 e 80 anos, sendo 24 mulheres e 25 homens. A maioria dos pacientes utilizavam mais que 5 medicamentos simultaneamente, obtendo uma média de 8 medicamentos por paciente. Todos os pacientes analisados são hipertensos e $28 \%$ também possuem diabetes. Dentre os pacientes avaliados, $78 \%$ dos prontuários apresentaram potenciais IMs, com um total de 123 interações; observou-se a ocorrência de IMs graves, embora a maior parte das IMs sejam classificadas como moderadas. Pacientes hipertensos apresentaram maior número de ocorrência de IMs que os pacientes hipertensos e diabéticos. CONCLUSÃO: Os resultados mostram que os pacientes analisados apresentam um número significativo de IMs. Diante desses resultados nota-se a necessidade da conscientização dos profissionais da saúde quanto à possível ocorrência destas, e um estudo mais aprofundado sobre as consequências das IMs em pacientes de hemodiálise.
\end{abstract}

Palavras -chave: Medicamentos. Hemodiálise. Hipertensão. Diabetes. Insuficiência Renal.

\begin{abstract}
:
INTRODUCTION:Chronic Diseases Kidney (CKD) patients to use a wide range of drugs, increasing the occurrence of possible drug-drug interactions (DDI). OBJECTIVE: Identify potential DDIs in the records from stage 5 patients with CKD, undergoing dialysis treatment, classifying them as their severity and separating its occurrence as the comorbidities of the patient.METHODOLOGY: The study was conducted in the Barra do Garças city, state of MatoGrosso, from July to December 2014. Then, it was analyzed the medical records of level 5 patients with CKD, who are undergoing dialysis treatment in this city. To identify possible DDIs, it was consulted Micromedex ${ }^{\circledR}$, a software, which has an interactive system to check its occurrence. RESULTS: The records of 49 patients were analyzed. The patients are aged between 25 and 80 years; 24 women and 25 men. Most of the patients used more than 5 drugs simultaneously, achieving an average of 8 medications per patient. All patients were hypertensive, and $28 \%$ of them also display diabetes. Among them, 78\% of the showed potential DDIs, with 123 interactions. Furthermore, it was observed the occurrence of serious DDIs, although most of the them were classified as moderate. Hypertensive patients had higher frequencies of DDIs occurrence in comparison to hypertensive and diabetic patients. CONCLUSION: The results emphasize that these patients have a significant number of DDIs. Based on these results, we observed the necessity for awareness the health professionals about the possible occurrence of these DDIs, and further studies on the consequences of them in hemodialysis patients.
\end{abstract}

Keywords: Medicines. Hemodialysis.Hypertension.Diabetes. Renal Insufficiency. 


\section{INTRODUÇÃO}

A Doença Renal Crônica (DRC) é um importante problema de saúde mundial, sendo que a doença possui uma incidência e prevalência avançada, com desfechos desfavoráveis e um alto custo no tratamento. A DRC é caracterizada pela lesão do parênquima renal ou pela diminuição da taxa de filtração glomerular (TFG) presentes por um período de três ou mais meses(BASTOS; BREGMAN; KIRSZTAJN, 2010).

A TFG pode diminuir drasticamente, chegando a casos avançados,nos quais a filtração glomerular decresce para 10 a 5 $\mathrm{ml} / \mathrm{min} / 1,73 \mathrm{~m}^{2}$, caracterizando o estágio 5 , ou seja, o último estágio da DRC. Nesse estágio é necessário que o paciente realize um transplante renal, ou inicie uma terapia de substituição da função renal, das quais a principal é a hemodiálise(DRAIBE; AJZEN, 2002; KDIGO, 2012).

Em 2013 a Sociedade Brasileira de Nefrologia (SBN) estimou que mais de cem mil pessoas utilizasse o tratamento dialítico, sendo que $90 \%$ desses pacientes realizavam o tratamento de hemodiálise (SOCIEDADE BRASILEIRA DE NEFROLOGIA, 2013).

A maioria dos pacientes com DRC possuem hipertensão arterial e Diabetes mellitus, sendo que estas duas patologias são consideradas as principais causas da DRC nos dias atuais(NASRI; RAFIEIAN-KOPAEI, 2015). A presença dessas comorbidades leva o paciente a fazer uso de uma ampla gama de medicamentos, concomitantemente com o tratamento dialítico. Pacientes portadores de DRC apresentam dentre outras complicações, problemas relacionados com a medicação, entre eles as interações medicamentosas (IM)s. As IMs podem interferir no resultado terapêutico desejado, muitas vezes de forma negativa na morbidade, mortalidade e qualidade de vida desses pacientes(MASON; BAKUS, 2010).

$\mathrm{O}$ uso de vários fármacos de forma concomitante é cada vez mais complexo e potencialmente prejudicial, levando ao paciente realizar um maior número de consultas médicas e hospitalizações. Os pacientes que utilizam vários medicamentos precisam de um acompanhamento regular, com a revisão e otimização das prescrições(GALLAGHER P, BARRY P, O, 2007; GUTHRIE et al., 2015).

O objetivo dessa pesquisa é identificar IMs, potencialmente prejudiciais em portadores de DRC no estágio 5 que realizam tratamento de hemodiálise, classificando-as quanto a sua gravidade e separando sua ocorrência quanto as comorbidades de cada paciente.

\section{METODOLOGIA}

Trata-se de um estudo farmacoepidemiológico do tipo quantitativo, seguindo o delineamento descritivo e transversal. $\mathrm{O}$ estudo foi realizado no município de Barra do Garças -MT, 
localizada na região da Amazônia Legal. Participaram da pesquisa 49 pacientes portadores de DRC no estágio 5 que realizavam tratamento de hemodiálise no Instituto de Nefrologia do Araguaia (INA).

Os dados clínicos e sóciodemográficos dos pacientes foram coletados por meio de prontuários físicos e pelo SoftwareNefrodata ACD, no qual ficam armazenadas todas as informações, inclusive todos os medicamentos de uso contínuo dos pacientes. Nesta etapa traçou-se o perfil farmacoterapêutico dos pacientes.

Para checar a ocorrência das possíveis IMs utilizou-se a base de dados MICROMEDEX ${ }^{\circledR}$ Thomson Midromedex ${ }^{\circledR}$ Health Series Software interativo com acesso on-line, através do portal de periódicos da Coordenação de Aperfeiçoamento de Pessoal de Nível Superior (CAPES).

Foram incluídos neste estudo pacientes com DRC no estágio5, que realizavam hemodiálise e que residiam na região do Médio Araguaia.

Os aspectos éticos e legais foram contemplados, em conformidade com a resolução n ${ }^{\circ}$. 466/2012 do Conselho Nacional de Saúde (CNS), que regulamenta pesquisas envolvendo seres humanos (Certificado de aprovação ética: 34568114.9.0000.5587).

\section{RESULTADOS}

$\mathrm{Na}$ primeira etapa, foi analisado o perfil dos pacientes com DRC participantes dessa pesquisa, foram analisados 49 prontuários, onde $51 \%$ pertenciam ao sexo masculino e $49 \%$ ao sexo feminino. Em relação à raça, $66 \%$ dos pacientes declaram-se de raça parda, $18 \%$ branca e $16 \%$ negra. A média de idade dos pacientes foi de 52 anos, sendo o paciente mais jovem de 25 anos, e o mais idoso de 80 anos. Quanto à escolaridade, $71 \%$ dos pacientes eram alfabetizados e $29 \%$ declaram-se analfabetos. Quanto ao diagnóstico clínico, $72 \%$ dos pacientes eram hipertensos e $28 \%$ eram tanto hipertensos, quanto diabéticos (tabela 1).

Tabela 1. Perfil demográfico e clínico dos pacientes com DCR- estágio 5.

\begin{tabular}{|c|c|c|}
\hline Características & $\mathbf{N}$ & $(\%)$ \\
\hline \multicolumn{3}{|l|}{ Gênero } \\
\hline Feminino & 24 & $49 \%$ \\
\hline Masculino & 25 & $51 \%$ \\
\hline \multicolumn{3}{|l|}{ Raça } \\
\hline Parda & 32 & $66 \%$ \\
\hline Branca & 9 & $18 \%$ \\
\hline Negra & 8 & $16 \%$ \\
\hline \multicolumn{3}{|l|}{ Idade } \\
\hline$<$ ou igual a 39 & 8 & $16 \%$ \\
\hline 40 a 59 & 24 & $49 \%$ \\
\hline$>$ ou igual a 60 & 17 & $35 \%$ \\
\hline \multicolumn{3}{|l|}{ Escolaridade } \\
\hline Analfabeto & 14 & $29 \%$ \\
\hline Ensino fundamental incompleto & 21 & $43 \%$ \\
\hline Ensino fundamental completo & 6 & $12 \%$ \\
\hline Ensino médio & 7 & $14 \%$ \\
\hline Ensino Superior & 1 & $2 \%$ \\
\hline \multicolumn{3}{|l|}{ Doenças Relacionadas } \\
\hline Hipertensão & 35 & $72 \%$ \\
\hline Hipertensão e Diabetes mellitus & 14 & $28 \%$ \\
\hline
\end{tabular}

Fonte: Dados dos autores 
$\mathrm{Na}$ avaliação do perfil farmacoterapêutico dos pacientes renais crônicos, foram listados um total de 418 medicamentos utilizados, sendo que eles possuem 60 princípios ativos diferentes, pertencentes a 27 classes terapêuticas distintas. As classes de medicamentos mais prescritas foram dos anti-hipertensivos $(34,7 \%)$, os hipoglicemiantes $(10,2 \%)$ e as vitaminas $(10,2 \%)$.

Os dez medicamentos mais prescritos foram o cloridrato de sevelamer (100\%), ácido fólico $(81,6 \%)$, ácido acetilsalicílico $(81,6 \%)$, complexo B $(73,5 \%)$, carbonato de cálcio $(61,2 \%)$, vitamina C $(59,2 \%)$, losartana potássica (49,0\%), nifedipino $(30,6 \%)$, furosemida $(24,5 \%)$ e hidroclorotiazida $(22,5 \%)$.

Entre os pacientes que apresentavam diagnóstico exclusivo para hipertensão arterial, foram listados o uso de um total de 290 medicamentos, dentre eles 53 princípios ativos diferentes e pertencentes a 23 classes terapêuticas distintas. Os medicamentos mais utilizados pelos pacientes hipertensos foram: o cloridrato de sevelamer (100\%), ácido acetilsalicílico $(85,7 \%)$, ácido fólico $(82,8 \%)$, complexo B $(77,2 \%)$ Vitamina C $(62,8 \%)$, carbonato de cálcio $(60,0 \%)$, losartana potássica $(51,4 \%)$, nifedipino $(28,6 \%)$, hidroclorotiazida $(20,0 \%)$ e a furosemida $(14,3 \%)$.

Dentre os pacientes que eram portadores tanto da hipertensão arterial, quanto de Diabetesmellitus, foram listados um total de 128 medicamentos, dentre eles 38 princípios ativos diferentes e pertencentes a 12 classes terapêuticas distintas. Os medicamentos mais utilizados por esses pacientes foram: o cloridrato de sevelamer(100\%), ácido fólico $(85,7 \%)$, ácido acetilsalicílico $(71,4 \%)$, complexo B $(64,3 \%)$, carbonato de cálcio $(64,3 \%)$, vitamina C $(50,0 \%)$, furosemida $(50,0 \%)$, losartana potássica $(42,8 \%)$, nifedipino $(28,6 \%)$ e omeprazol $(28,6 \%)$.

Os pacientes foram classificados quanto a quantidade de medicamentos utilizados. A maioria dos pacientes utiliza mais que 5 medicamentos simultaneamente, obtendo uma média de 8 medicamentos por paciente. Ao avaliarmos pacientes com diagnóstico de hipertensão, $66 \%$ deles utilizam entre 5-8 medicamentos, enquanto que os pacientes portadores de hipertensão e Diabetes mellitus, em sua maioria (64\%), utiliza 9 ou mais medicamentos (Figura1).

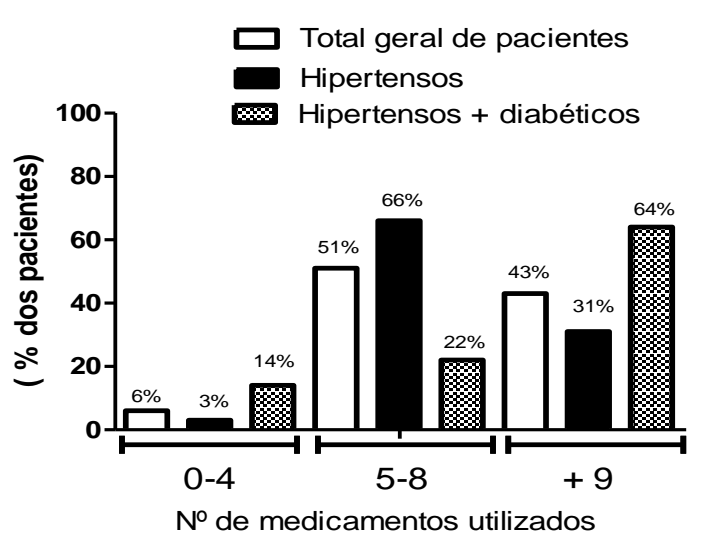

Figura 1- Quantidade de medicamentos utilizados pelos pacientes em hemodiálise. 
$\mathrm{Na}$ segunda etapa foi realizado a análise dos medicamentos através da base de dadosMICROMEDEX ${ }^{\circledR}$

Thomson

Midromedex ${ }^{\circledR}$ Health Series Software, onde foram identificadas 123 potenciais IMs, sendo elas 66 IMs distintas, presentes em 78\% dos prontuários. Sendo assim, $86 \%$ dos prontuários dos pacientes hipertensos e $57 \%$ dos prontuários de pacientes hipertensos e diabéticos apresentaram algum tipo de IMs (Figura 2).

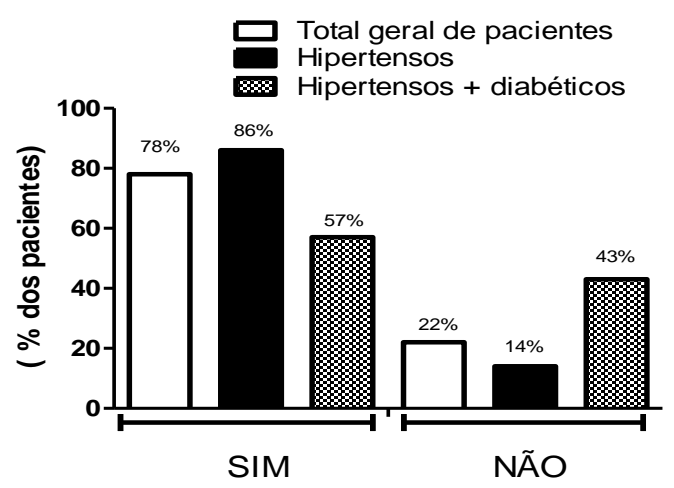

Figura 2- Ocorrência de IMs identificadas nos prontuários dos pacientes.

A figura 3 e a tabela 2 demonstram a gravidade das IMs observadas nos prontuários de cada grupo de pacientes. De uma forma geral, ocorram mais IMs moderadas, tanto em pacientes hipertensos, como em pacientes hipertensos e diabéticos. A distribuição geral de IMs leves, moderadas e graves se deram de forma similar entre todos os pacientes. $\mathrm{Na}$ tabela 2, estão descritas as IMs que ocorram com mais frequência entre os pacientes com DCR.

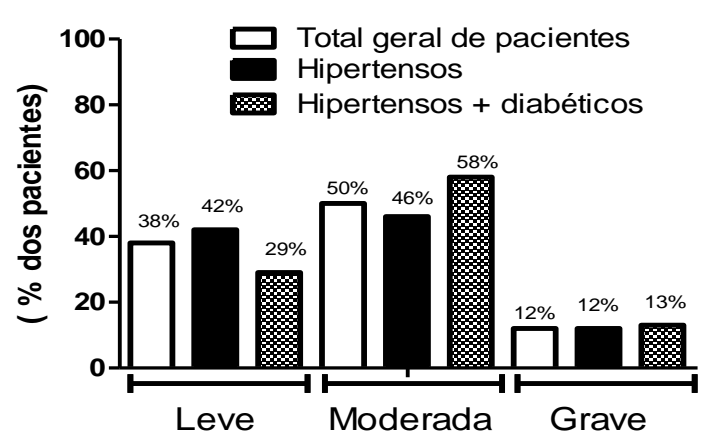

Figura 3- Classificação das IMs quanto a sua gravidade.

\section{DISCUSSÃO}

Os principais resultados encontrados em nosso estudo foram: (a) a maior parte dos pacientes são hipertensos e (b) fazia uso de mais de 5 medicamentos concomitantemente; (c) a maioria dos prontuários apresentaram IMs, sendo que (d) observou-se a ocorrência de IMs graves, embora a maior parte das IMs seja classificadas como moderadas; (e) pacientes hipertensos apresentam maior número de ocorrência IMs que os pacientes hipertensos e diabéticos; (f) a IM de ocorrência mais frequente foi entre o ácido ascórbico e a cianocobalamina sendo considerada, uma IM menor.

Todos os pacientes analisados são hipertensos e $28 \%$ deles são também diabéticos. De acordo com as Diretrizes Brasileiras de Hipertensão Arterial, a DRC é uma das complicações mais frequentes ocasionada pela hipertensão arterial. A hipertensão também pode ser uma complicação da doença renal, pois os rins são essenciais na regulação em longo prazo da pressão sanguínea(NOBRE et al., 2010). 
Tabela 2. Duplas de medicamentos com interações mais frequentes, classificados de acordo com a gravidade, em pacientes com DRC no estágio 5.

\begin{tabular}{|c|c|c|c|}
\hline $\begin{array}{l}\text { Medicamento } 1 \text { x Medicamento } 2 \\
\text { Classificação da interação } \\
\text { Ocorrência n }(\%)\end{array}$ & Descrição & Mecanismo Provável & Gestão clínica \\
\hline $\begin{array}{c}\text { Ácido ascórbico x Complexo B } \\
\text { IM Leve } \\
26(21,13 \%)\end{array}$ & $\begin{array}{l}\text { Quantidades reduzidas de } \\
\text { cianocobalamina disponível. }\end{array}$ & Desconhecido & $\begin{array}{l}\text { O ácido ascórbico deve ser administrado duas ou mais } \\
\text { horas após uma refeição ou suplementos de vitamina } \\
\text { B12. }\end{array}$ \\
\hline $\begin{array}{l}\text { Complexo B x Omeprazol } \\
\text { IM Leve } \\
6(4,87 \%)\end{array}$ & $\begin{array}{l}\text { Absorção diminuída da } \\
\text { cianocobalamina. }\end{array}$ & pH gástrico alterado & $\begin{array}{l}\text { Se possível, mudar para outra medicação anti-úlcera e } \\
\text { separar as doses por pelo menos duas horas, ou } \\
\text { cianocobalamina por via intramuscular ou subcutânea } \\
\text { seria preferido. }\end{array}$ \\
\hline $\begin{array}{l}\text { Carbonato decálcio x Sulfato ferroso } \\
\text { IM Leve } \\
4(3,25 \%)\end{array}$ & Diminuição da eficácia de ferro. & $\begin{array}{l}\text { Diminuição da absorção de } \\
\text { ferro. }\end{array}$ & $\begin{array}{l}\text { Se o uso concomitante não pode ser evitado, sais de ferro } \\
\text { devem ser tomados pelo menos uma hora antes ou duas } \\
\text { horas após alumínio, cálcio ou magnésio. }\end{array}$ \\
\hline $\begin{array}{c}\text { Atenolol x Carbonato de cálcio } \\
\text { IM Leve } \\
4(3,25 \%)\end{array}$ & Eficácia reduzida de atenolol. & $\begin{array}{l}\text { Diminuição da absorção } \\
\text { atenolol. }\end{array}$ & $\begin{array}{l}\text { Atenolol deve ser administrado duas horas antes ou seis } \\
\text { horas após o alumínio, de cálcio ou de magnésio que } \\
\text { contêm o produto. }\end{array}$ \\
\hline $\begin{array}{c}\text { Carbonato de cálcio } \mathrm{x} \\
\text { Hidroclorotiazida } \\
\text { IM moderada } \\
4(3,25 \%)\end{array}$ & $\begin{array}{l}\text { Aumento do risco de } \\
\text { hipercalcemia. }\end{array}$ & $\begin{array}{l}\text { Inibição da excreção renal do } \\
\text { cálcio. }\end{array}$ & $\begin{array}{l}\text { Considere o nível de cálcio monitoramento soro e / ou } \\
\text { função da paratireoide. }\end{array}$ \\
\hline $\begin{array}{l}\text { Furosemida x Insulina } \\
\text { IM Moderada } \\
3(2,43 \%)\end{array}$ & $\begin{array}{l}\text { Aumento do risco de } \\
\text { hiperglicemia. }\end{array}$ & $\begin{array}{l}\text { Metabolismo da glicose } \\
\text { alterada. }\end{array}$ & $\begin{array}{l}\text { Pode ser necessário ajuste da dose de agentes } \\
\text { antidiabéticos orais. }\end{array}$ \\
\hline $\begin{array}{c}\text { Espironolactona x Losartana Potássica } \\
\text { IM Moderada } \\
3(2,43 \%)\end{array}$ & Pode resultar em hipercalemia. & $\begin{array}{l}\text { Retenção de aditivo de } \\
\text { potássio. }\end{array}$ & $\begin{array}{l}\text { Monitorar os níveis de potássio sérico durante o } \\
\text { tratamento. }\end{array}$ \\
\hline $\begin{array}{c}\text { Besilato de anlodipino x Prednisona } \\
\text { IM Moderada } \\
3(2,43 \%)\end{array}$ & $\begin{array}{l}\text { Redução da eficácia de } \\
\text { amlodipina. }\end{array}$ & $\begin{array}{l}\text { Indução do metabolismo } \\
\text { mediado por CYP3A4 de } \\
\text { amlodipina de prednisona. }\end{array}$ & A pressão arterial deve ser monitorada. \\
\hline $\begin{array}{c}\text { Complexo B x Sinvastatina } \\
\text { IM Grave } \\
4(3,25 \%)\end{array}$ & Aumento da niacina e sinvastatina & Desconhecido & $\begin{array}{c}\text { Monitorar o paciente para sinais e sintomas de miopatia } \\
\text { ou rabdomiólise. }\end{array}$ \\
\hline $\begin{array}{c}\text { Besilato de Anlodipino x Sinvastatina } \\
\text { IM Grave } \\
2(1,62 \%)\end{array}$ & $\begin{array}{l}\text { Aumento da exposição } \\
\text { sinvastatina }\end{array}$ & Desconhecido & A dose de sinvastatina não deve exceder $20 \mathrm{mg} /$ dia. \\
\hline
\end{tabular}

Fonte: MICROMEDEX ${ }^{\circledR}$ Thomson Midromedex ${ }^{\circledR}$ Health Series 
O diabetes também é um fator de risco da DRC, conhecida como nefropatia diabética, aproximadamente $35 \%$ dos pacientes diabéticos desenvolvem a doença renal (DWYER et al., 2012).

Em nosso estudo a maioria dos pacientes utilizava mais de cinco medicamentos, sendo a média de oito medicamentos utilizados por paciente, esses resultados se equiparam ao estudo de Sgnaolin\& colaboradores (2014)(SGNAOLIN et al., 2014), onde foi identificada uma média de aproximadamente seis medicamentos utilizados por pacientes. Um outro estudo realizado por Sontakkeet al (2015), também demonstrou que a média de medicamentos utilizados pelos pacientes renais foram de oito medicamentos por paciente, acredita-se que a utilização de oito fármacos ou mais, pode potencializar em $100 \%$ a ocorrência de IMs(SONTAKKE et al., 2015).

$\mathrm{O}$ alto número de medicamentos utilizados pelos pacientes renais se deve às comorbidades presentes na doença renal e as inúmeras complicações da doença. Entre as complicações mais comuns apresentadas pelos pacientes renais estão as doenças cardiovasculares, a anemia, alterações do metabolismo mineral, acidose metabólica e dislipidemias(BASTOS;

BREGMAN; KIRSZTAJN, 2010).

Foram encontradas possíveis IMs na maior parte dos prontuários, fatores como idade, presença de comorbidades, quantidade de medicamentos utilizados e quantidade de médicos que cuidam de um paciente podem potencializar a ocorrência de IMs. A maioria dessas interações apresentaram gravidade moderada. Outros estudos com pacientes de DRC, também encontraram resultados semelhantes, como no estudo conduzido em um ambulatório em Juiz de Fora, onde foram identificadas IMs moderadas na maioria dos prontuários(BATISTA et al., 2013). Em pacientes com DRC, a ocorrência de problemas relacionados aos medicamentos, como as interações, é muito comum devido a grande quantidade de medicamentos utilizados por esses pacientes(MASON; BAKUS, 2010).

Apesar de as IMs moderadas não apresentarem o risco maior aos pacientes, essas não devem ser ignoradas, é sabido que elas podem agravar o estado de saúde do paciente, em alguns casos exigindo uma mudança no tratamento (MICROMEDEX).

A IM moderada que ocorreu com uma frequência maior, foi entre os medicamentos carbonato de cálcio (repositor hidroeletrolítico) e a hidroclorotiazida (antihipertensivo diurético) (MICROMEDEX). Essa interação pode resultar na inibição da excreção renal do cálcio levando a quadros de hipercalcêmica. Como a excreção no paciente renal já é prejudicada, essa interação poderia potencializar o aumento desse cálcio no organismo, o que acarretaria em mais uma complicação para estes pacientes. 
As interações graves ocorreram em $18 \%$ dos prontuários, sendo 13 duplas de medicamentos distintos, um total que representa quase um quarto das IMs identificadas. Um estudo realizado em um hospital terciário no Sul da Índia, também encontro resultados parecidos com $20 \%$ das IMs graves. As IMs graves podem por em risco a vida do paciente e causar graves efeitos adversos, essas interações requerem intervenção médica para a diminuição dos prejuízos que podem ser causados ao paciente(RAMA et al., 2012).

Nesse estudo a interação grave que ocorreu mais vezes foi entre o complexo B e a sinvastatina, essa interação pode resultar num aumento da exposição à sinvastatina, podendo levar o paciente a desenvolver problemas como miopatia ou rabdomiólise (MICROMEDEX). Esses pacientes necessitam de um cuidado maior e a monitoração frequente para os sinais e sintomas dessas patologias. Em pacientes renais, onde a excreção renal de fármacos é prejudicada essa monitoração do paciente deve ocorrer de forma ainda mais frequente.

No estudo indiano, a dupla de medicamentos que interagiu com mais frequência foi o ácido ascórbico (Vitamina C) e a cianocobalamina (Vitamina B12), esse achado também foi encontrado em nosso estudo, sendo essa IM considerada de gravidade menor e foi identificada em 26 prontuários (RAMA et al., 2012).
A anemia é uma complicação comum na doença renal crônica, uma das causas da anemia é a carência de vitamina B12, essa vitamina auxilia na formação das células vermelhas do sangue. A vitamina B12 pode ser usada como adjuvante no tratamento da anemia no caso de pacientes em apresentem carência dessa vitamina. Já a vitamina C auxilia na absorção do ferro, porem no paciente renal não é necessário uma que se faça uma suplementação frequente no tratamento da anemia. A anemia quando não tratada pode causar muito prejuízos ao paciente renal, como internações mais frequentes, diminuição na qualidade de vida e maior taxa de mortalidade (ABENSUR, 2009).

Os pacientes que utilizam mais de 9 medicamentos e possuíam as duas comorbidades associadas, a hipertensão e o diabetes, apresentaram um menor número de IMs. Sabe-se que quanto maior o número de medicamentos utilizados, e patologias presente, maior é a probabilidade de ocorrência de IMs. Associa-se esse achado ao fato de nos prontuários dos pacientes que eram apenas hipertensos ocorrerem uma maior diversidade de princípios ativos, nos prontuários dos pacientes hipertensos foram encontrados 53 princípios ativos distintos e 23 classes terapêuticas diferentes, enquanto que nos pacientes hipertensos e diabéticos foram encontrados 38 princípios ativos diferentes e apenas 12 classes terapêuticas distintas. 
Os pacientes que são apenas hipertensos fazem uso de medicamentos de classes como a dos antiarrítmicos, cardiotônicos digitálicos (digoxina) e antidepressivos, esses medicamentos estão frequentemente envolvidos na ocorrência de potenciais IMs (AMARAL; PERASSOLO, 2012; LOCATELLI, 2007).

Porém vale ressaltar que os pacientes hipertensos e diabéticos, apesar de apresentarem menor quantidade de IMs, apresentaram mais IMs classificadas como graves, que são interações que causam risco a vida do paciente.

\section{CONSIDERAÇÕES FINAIS}

Os achados nesse estudo mostraram que os pacientes em hemodiálise na sua maioria fazem uso de aproximadamente oito medicamentos, o que potencializa a ocorrência de IMs, principalmente IMs moderadas. Também mostraram que mesmo os pacientes que fazem uso de uma menor quantidade de medicamentos, podem utilizar fármacos com maior potencial de IMs.

Esses resultados evidenciam a necessidade de uma equipe multiprofissional capacitada, capaz de identificar essas potenciais interações, para colaborar com a eficiência terapêutica do tratamento, para que se possa diminuir as injurias causadas por essas interações, sabendo-se que muitas destas interações prejudicam de forma fatal a vida desses pacientes.
Também mostra a necessidade de um estudo aprofundado sobre as consequências dessas interações em pacientes em tratamento de hemodiálise.

\section{REFERÊNCIAS BIBLIOGRÁFICAS}

ABENSUR, H. Diagnóstico e tratamento da anemia na DRC. Jornal Brasileiro de Nefrolologia, v. 31, n. 2, p. 76-77, 2009.

AMARAL, D. M. D.; PERASSOLO, M. S. Possíveis interações medicamentosas entre os anti- hipertensivos e antidiabéticos em participantes do Grupo HIPERDIA de Parobé , RS ( Uma análise teórica ). journal of basic and applled pharmaceutical sciences, v. 33, n. 1, p. 99-105, 2012.

BASTOS, M. G.; BREGMAN, R.; KIRSZTAJN, G. M. Doença renal crônica: frequente e grave, mas também prevenível e tratável. Revista da Associação Médica Brasileira, v. 56, n. 2, p. 248-253, 2010. BATISTA, A. et al. Interações medicamentosas potenciais em pacientes com doença renal crônica. Jornal Brasileiro de Nefrologia, v. 36, n. 1, p. 26-34, 2013.

DRAIBE, S. A.; AJZEN, H. Insuficiência renal crônica. N. Nefrologia: guia de medicina ambulatorial, n. Tabela 2, p. 339343, 2002.

DWYER, J. P. et al. Renal Dysfunction in the Presence of Normoalbuminuria in Type 2 Diabetes: Results from the DEMAND Study. Cardiorenal Medicine, v. 2, n. 1, p. 1-10, 2012.

GALLAGHER P, BARRY P, O, M. D. Inappropriate prescribing in the elderly. Journal of Clinical Pharmacy and Therapeutics, v. 32, p. 113-121, 2007. 
GUTHRIE, B. et al. The rising tide of polypharmacy and drug-drug interactions : population database analysis $1995-2010$.

BMC Medicine, v. 13:74, p. 1-10, 2015.

Kidney Disease: Improving Global Outcomes (KDIGO) CKD WORK GROUP. KDIGO 2012 Clinical Practice Guideline for the Evaluation and Management of Chronic Kidney DiseaseKidney International Supplements. [s.l: s.n.]. Disponível em: $<$ http://www.kdigo.org/clinical_practice_guid elines/pdf/CKD/KDIGO CKD-MBD GL KI Suppl113.pdflnhttp://www.nature.com/doifin der/10.1038/kisup.2012.73 \nhttp://www.natur e.com/doifinder/10.1038/kisup.2012.76>.

KLASCO, R. K.(Ed). DRUG-REAX System . Thomson MICROMEDEX, Greenwood Village, Colorado, USA; c1974-2012. Disponível em: http://www.thomsonhc.com. LOCATELLI, J. Interações medicamentosas em idosos hospitalizados Drug interactions in hospitalized elderly patients. einstein, v. 5, n. 4, p. 343-346, 2007.

MASON, N. A.; BAKUS, J. L. Strategies for Reducing Polypharmacy and Other Medication-Related Problems in Chronic Kidney Disease. Seminars in Dialysis, v. 23, n. 1, p. 55-61, jan. 2010.

NASRI, H.; RAFIEIAN-KOPAEI, M.

Diabetes mellitus and renal failure:

Prevention and management. Journal of research in medical sciences : the official journal of Isfahan University of Medical Sciences, v. 20, n. 11, p. 1112-20, nov. 2015. NOBRE, F. et al. VI Diretrizes Brasileiras de Hipertensão. Arquivos Brasileiros de

Cardiologia, v. 95, n. 1, p. 1-51, 2010.

RAMA, M. et al. Assessment of Drug-Drug Interactions among Renal Failure Patients of Nephrology Ward in a South Indian Tertiary
Care Hospital. Indian journal of pharmaceutical sciences, v. 74, n. 1, p. 63-8, jan. 2012.

SGNAOLIN, V. et al. Avaliação dos medicamentos utilizados e possíveis interações medicamentosas em doentes renais crônicos. Scientia Medica., v. 24, n. 4, p. 329-335, 2014.

Sociedade Brasileira de Nefrologia (SBN).Censo dos Centros de Diálise no Brasil - 2013. Sociedade Brasileira de Nefrologia. Disponível em http:<//www.sbn.org.br>. Acesso em: junho de 2014.

SONTAKKE, S. et al. Evaluation of adherence to therapy in patients of chronic kidney disease. Indian journal of pharmacology, v. 47, n. 6, p. 668-71, 2015. 